\title{
Computer-aided detection of lung nodules on multidetector row computed tomography using three-dimensional analysis of nodule candidates and their surroundings
}

\author{
Sumiaki Matsumoto • Yoshiharu Ohno \\ Hitoshi Yamagata · Daisuke Takenaka \\ Kazuro Sugimura
}

(C) Japan Radiological Society 2009

Erratum to: Radiat Med (2008) 26:562-569

DOI 10.1007/s11604-008-0272-5

During correction of the above mentioned article, error was inadvertently introduced.

In Abstract, Purpose should be partly revised as follows.

Purpose. We have been developing a computer-aided detection (CAD) system for lung nodules on multidetector row computed tomography (MDCT). The scheme for nodule detection in this system is featured by threedimensional analysis of nodule candidates and their surroundings, which is designed to discriminate nodules from blood vessels. The purpose of this study was to evaluate the CAD system.

The online version of the original article can be found under doi 10.1007/s11604-008-0272-5

S. Matsumoto $(\bowtie) \cdot$ Y. Ohno $\cdot$ D. Takenaka $\cdot$ K. Sugimura Department of Radiology, Kobe University Graduate School of Medicine, 7-5-2 Kusunoki-cho, Chuo-ku, Kobe 650-0017, Japan Tel. +81-78-382-6104; Fax +81-78-382-6129

e-mail: sumatsu@med.kobe-u.ac.jp

H. Yamagata

Research and Development Center, Toshiba Medical Systems

Corporation, Otawara, Japan 